\title{
Inflammatory pseudotumor of Castleman disease and lgG4-related disease masquerading as kidney malignancy
}

\author{
Bolong Liu' ${ }^{1 \dagger}$, Yong Huang ${ }^{1 \dagger}$, Luying Tang ${ }^{2}$, Jiexia Guan², Xiangfu Zhou ${ }^{1 *}$ and Hailun Zhan ${ }^{1 *}$
}

\begin{abstract}
Background: With widespread clinical application of imaging techniques, renal space-occupying lesions have been identified at an increasing frequency. Here, we report two rare cases, Castleman disease (CD) and IgG4-related disease (IgG4-RD), presenting primarily with the symptoms and imaging findings of kidney malignancy.

Case presentation: In case 1, an occupying lesion located in the right renal pelvis was detected using magnetic resonance imaging in a 32-year-old female who presented with hematuria and lumbago. First misdiagnosed as carcinoma of the renal pelvis, the patient underwent right radical nephroureterectomy. However, postoperative pathological and immunohistochemistry studies finally confirmed the diagnosis of CD. In case 2, a 45-year-old male presented with the chief complaint of anuria. Nephrostomy and renal biopsy indicated lymphoma, following which, antegrade urography and computed tomography urography were performed, which revealed bilateral hydronephrosis and mass lesions around the renal pelvis. Partial resection of the masses and frozen section examination indicated the diagnosis of CD. However, the results of postoperative histopathology and immunohistochemistry combined with serum lgG4 were consistent with IgG4-RD. Both the patients recovered well after drug treatment without recurrence of the diseases.
\end{abstract}

Conclusions: Inflammatory pseudotumor of CD and IgG4-RD with kidney involvement are primarily diagnosed by postoperative histopathology and can pose a preoperative diagnostic challenge because these lesions can masquerade as kidney malignancy. Therefore, we recommend core biopsy as a nonnegligible procedure to evaluate renal masses and potentially prevent unnecessary surgical treatment.

Keywords: Kidney malignancy, Castleman disease, IgG4-related disease, Inflammatory pseudotumor

\section{Background}

With widespread clinical application of computed tomography (CT), magnetic resonance imaging (MRI), ultrasound, and other imaging technologies, renal spaceoccupying lesions have been detected at an increased frequency. Renal space-occupying lesions include common diseases of the urinary system. The most frequently

\footnotetext{
*Correspondence: zhouxfu@mail.sysu.edu.cn; hailunzhan125@163.com ${ }^{\dagger}$ Bolong Liu and Yong Huang contributed equally to this work.

'Department of Urology, the Third Affiliated Hospital of Sun Yat-Sen University, Guangzhou, China

Full list of author information is available at the end of the article
}

encountered malignant renal masses in clinical practice are renal cell carcinoma, urothelial carcinoma, lymphoma, and metastasis, while the most common benign renal solid masses include angiomyolipoma, renal oncocytoma, and inflammatory pseudotumor [1]. However, making preoperative diagnoses remains a challenge owing to rare benign masses with atypical imaging findings, which is often confused with kidney malignancy. Among numerous highly unusual diseases, inflammatory pseudotumor of Castleman disease (CD) and IgG4related disease (IgG4-RD) are often misdiagnosed by clinicians as malignant neoplasms of the kidney. This 
can be attributed to their lack of typical or specific clinical manifestations and imaging characteristics, and their ability to manifest as renal lesions [2, 3].

$\mathrm{CD}$ is a rare lymphoproliferative disorder, primarily presenting as regional or generalized lymphadenopathy with multiorgan involvement. The disease can be classified into three histological variants, namely, hyaline vascular (HV) type, plasma cell (PC) type and a mixed form $[4,5]$. IgG4-RD is an emerging idiopathic immune-mediated disorder characterized by elevated serum IgG4 concentration and the presence of IgG4bearing lymphoplasmacytic infiltrates, resulting in chronic progressive inflammation accompanied by fibrosis and sclerosis in the affected organs $[6,7]$. Both diseases present with a broad spectrum of common clinical manifestations, with diagnosis principally dependent on pathological examination. Moreover, $\mathrm{CD}$ and IgG4-RD exhibit partially overlapping clinical and pathological features, as lymphadenopathy, infiltration by IgG4-positive plasma cells, and enhanced IgG4 levels in serum, complicating diagnosis of these conditions [8]. Inflammatory pseudotumor of $\mathrm{CD}$ or IgG4-RD, masquerading as kidney malignancy, can lead to inappropriate therapy or overtreatment, and may aggravate patients' conditions. Therefore, differential diagnosis of $\mathrm{CD}$ and IgG4-RD from kidney malignancy based on clinical manifestations, imaging, and pathological findings is essential. Here, we report one case each of $C D$ and IgG4-RD, presenting primarily with symptoms and imaging findings of kidney malignancy, admitted to our hospital between 2016 and 2019.

\section{Case presentation Case 1}

A 32-year-old female was referred to our urology clinic and presented with persistent, painless gross hematuria and intermittent, blunt lumbago for 15 days. She did not report any fever, fatigue, edema, dysuria, urgent or frequent urination. Physical examination findings and all laboratory values, including complete blood count, renal and liver functions, and coagulation profile were unremarkable, except for urine erythrocyte at 343.5 /ul (normal range within 0-26/ul). Urinary cytology and fluorescent in situ hybridization of exfoliated cells were both negative. However, contrast-enhanced abdominal MRI revealed an irregular mass, approximately $5.7 \times$ $3.5 \times 1.9 \mathrm{~cm}$ in size, in the right renal pelvis (Fig. $1 \mathrm{~A}$ ), with hypointensity on the T1 weighted image (Fig. 1B) and slight hyperintensity on the T2 weighted image (Fig. 1C). Meanwhile, the enhancement of the mass was markedly increased during the arterial phase (Fig. 1D) and decreased during the portal and delayed phases (Fig. 1E, F) with multiple enlarged lymph nodes (mild lymphadenectasis) in the right renal hilum. Based on the symptoms and imaging characteristics, a tentative diagnosis of carcinoma of the right renal pelvis was made.

Therefore, the patient underwent laparoscopic right radical nephroureterectomy with partial cystectomy and lymph node clearance around the renal pedicle. Intraoperatively, an elliptic solid tumor, approximately $6 \times 4 \mathrm{~cm}$ in size, with unclear boundary, was located in the right renal pelvis. The cut surface appeared grayish-white in color, and sectioning showed hard texture without hemorrhage and necrosis. Grossly, the mass partially invaded the adipose tissue of the renal sinus, excluding the perirenal fat (Fig. 2A). Postoperative pathology reported lymphoid tissue hyperplasia in the specimen and eosinophilic deposits were observed in the follicle centers with numerous plasma cells infiltrating the paracortex zone and lymphatic sinus (Fig. 2B-D). Immunohistochemical staining indicated CD38 (+), CD138 (+) in the interfollicular plasma cells (Fig. 2E, F). In summary, the pathology and immunohistochemistry supported the diagnosis of PC type CD.

Subsequently, the patient visited the hematology department for further treatment of CD. A bone marrow biopsy was performed, which excluded hematological malignancies. Serology testing for human herpesvirus-8 (HHV-8) and human immunodeficiency virus (HIV) were both negative. A positron emission tomography with computed tomography (PET/CT) scan revealed slightly elevated metabolism of the operative region $\left(\mathrm{SUV}_{\max } 2.7\right)$ and in multiple enlarged lymph nodes (SUV $_{\max }$ 2.4-3.3) including the parotid, neck, axilla, hilar and mediastinal nodes. Thereafter, she was treated with R-COP (rituximab $600 \mathrm{mg}$ day $1+$ cyclophosphamide 1.2 g day 2 + vindesine $4 \mathrm{mg}$ day 2 + prednisone $50 \mathrm{mg}$ day2day6) chemotherapy. Six courses later, the patient recovered, and symptoms disappeared. A follow-up PET/CT showed shrinkage and normal metabolism in the lymph nodes mentioned above with no recurrence.

\section{Case 2}

A 45-year-old male with a history of hyperuricemia was brought to the local hospital with chief complaint of anuria for 2 days, with no occurrence of fever, edema, lumbago, hematuria, pyuria, or lower abdominal pain. CT of the abdomen indicated bilateral hydronephrosis and tumors in the renal pelvis and serum creatinine rose to $400 \mu \mathrm{mol} / \mathrm{L}$ (normal range within $32-116 \mu \mathrm{mol} / \mathrm{L}$ ). Consequently, percutaneous ultrasound-guided nephrostomy was performed to relieve obstruction and an ultrasound-guided renal biopsy performed on both sides indicated lymphoma.

A few days later he presented to our hospital for further treatment. The antegrade urography demonstrated bilateral hydronephrosis and ureteropelvic junction obstruction with prominent filling defect of the renal 

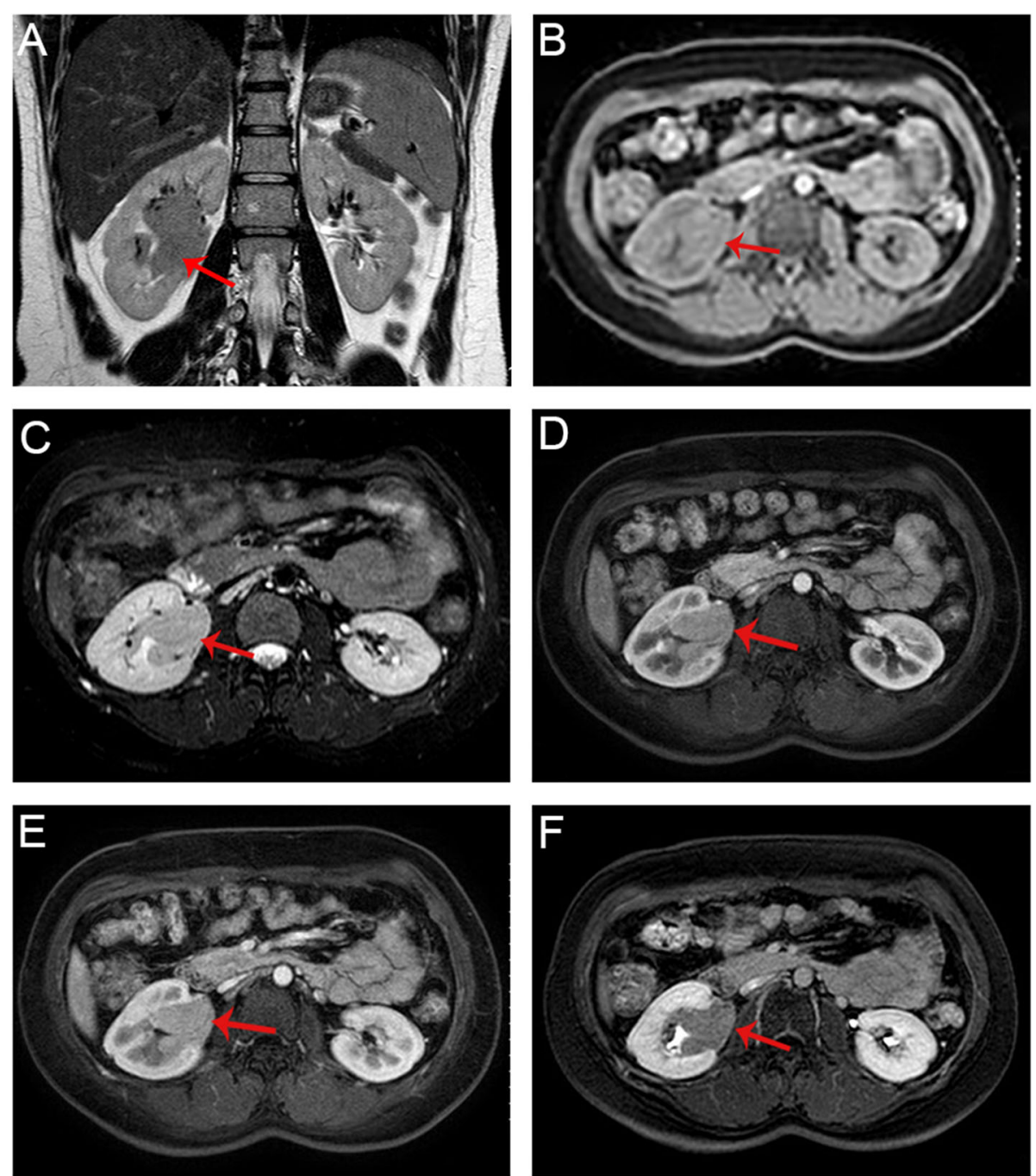

Fig. 1 Contrast-enhanced abdominal MRI of the lesion (red arrow) in case 1. (A) Coronal T2-weighted image; (B) Transverse T1-weighted with fat suppression (C) Transverse T2-weighted with fat-suppression; (D) Transverse T1-weighted with fat-suppression in arterial phase; (E) Transverse T1weighted with fat-suppression in portal phase; (F) Transverse T1-weighted with fat-suppression in delayed phase

pelvis (Fig. 3A, B). Meanwhile, physical examination revealed no significant abnormalities. His laboratories were notable for low hemoglobin $(113 \mathrm{~g} / \mathrm{L}$, with normal range within $130-175 \mathrm{~g} / \mathrm{L})$, high eosinophil count $\left(0.67 * 10^{9} / \mathrm{L}\right.$, with normal range within $0.02-0.52 * 10 \% / \mathrm{L})$, high creatinine $(146 \mu \mathrm{mol} / \mathrm{L}$, with normal range within $32-116 \mu \mathrm{mol} /$ $\mathrm{L})$, and high uric acid $(606 \mu \mathrm{mol} / \mathrm{L}$, with normal range within $90-420 \mu \mathrm{mol} / \mathrm{L}$ ). The glomerular filtration rate (GFR) of the left kidney was $14.5 \mathrm{ml} / \mathrm{min}$, and that of the right was $22.2 \mathrm{ml} / \mathrm{min}$. The subsequent contrast-enhanced CT urography (CTU) yielded soft-tissue density masses with an irregular border and mild-to-moderate homogeneous enhancement around the bilateral renal pelvis, measuring approximately $64 \times 48 \times 73 \mathrm{~mm}$ (right) and $74 \times 50 \times 74 \mathrm{~mm}$ (left). And the renal pelvis was compressed with a smooth surface. Obstruction at the ureteropelvic transition, with moderate dilatation and effusion of the renal pelvis and calyces was noticed. Multiple enlarged lymph nodes were observed in the lesser omental sac, hilum of liver and spleen, perirenal and paraaortic regions (Fig. 3C-F). Urine cytology did not reveal any cancer cells. Based on the above results, the patient was tentatively diagnosed with lymphoma preoperatively.

Intraoperative excisional biopsy and frozen section examination performed to identify the precise nature of the mass indicated a diagnosis of mixed-type $C D$ and ruled out malignancy. To clinically remove the obstruction and rapidly improve renal function, the patient underwent partial resection of the renal hilum masses on both sides. On gross examination, the specimen was composed of a solid mass of gray-yellow and gray-red 

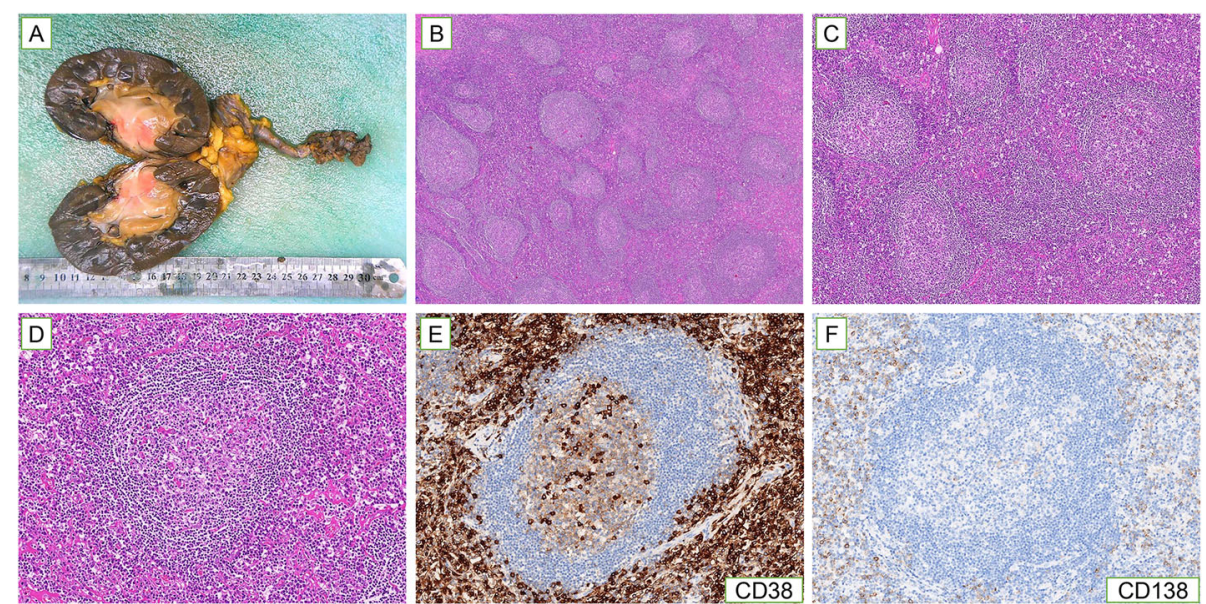

Fig. 2 Histopathologic features of the mass in case 1. (A) Gross observation of the mass in the right renal pelvis. (B) Abundant lymphoid tissue with lymphatic follicles in the specimen (40X). (C) Numerous plasma cells infiltrate the paracortex zone and lymphatic sinus (100x). (D) Eosinophilic deposits were observed in the follicle centers (200X). (E,F) Immunohistochemistry showed that the interfollicular area was infiltrated by CD38 (E) positive and CD138 (F) positive plasma cells (200X)

tissue measuring roughly $5 \times 4 \times 3 \mathrm{~cm}$. Sectioning of the tissue revealed hard texture (Fig. 4A). Histopathological examination revealed increased degree of fibrosis and dense infiltration of plasma cells and lymphocytes, with storiform-type fibrosis and obliterative phlebitis (Fig. 4BD). Immunohistochemistry demonstrated that many of the plasma cells were IgG $(+)$ and IgG4 $(+)$. More than $40 \%$ of the plasma cells were IgG4 $(+)$ with more than 10 IgG4 $(+)$ plasma cells per high powered field (HPF) of specimen (Fig. 4E, F). Serum IgG4 level was $27.40 \mathrm{~g} / \mathrm{L}$ (normal range within $0.03-2.01 \mathrm{~g} / \mathrm{L}$ ). Thus, the diagnosis of IgG4-RD was finally confirmed, at which time treatment with methylprednisolone $(40 \mathrm{mg} /$ day) and cyclophosphamide $(0.2 \mathrm{~g}$ every other day) was initiated, followed by a gradual decrease of the dose. Repeat CT scan 3 months later revealed no hydronephrosis, with significant reductions in the size of renal lesions and enlarged lymph nodes. Follow-up studies performed 9 months after the diagnosis confirmed a decrease in serum IgG4 and creatinine to $2.21 \mathrm{~g} / \mathrm{L}$ and $118 \mu \mathrm{mol} / \mathrm{L}$, respectively. No recurrence was observed as the patient was prescribed low dose methylprednisolone.

\section{Discussion and conclusions}

Our cases highlighted the imaging of renal spaceoccupying lesions and the symptoms related to presence of a renal mass, including painless gross hematuria, lumbago, and anuria. None of these symptoms directly indicate $\mathrm{CD}$ and IgG4-RD. However, these symptoms and imaging findings often raise high suspicion of kidney malignancy, frequently leading to misdiagnosis because of its increasing frequency and higher risk [9]. The widespread availability of abdominal CT, MRI, and ultrasound has led to an increase in the diagnosis of renal tumors. In 2018, there were more than 400,000 new cases of cancers of the kidney and renal pelvis globally, representing an estimated $2.2 \%$ of the global cancer burden, with more than 175,000 deaths due to this disease [10].

The diagnosis and management of kidney cancer have developed rapidly in recent years. The symptoms of kidney cancer include the classic clinical triad of hematuria, flank pain and palpable mass, as well as paraneoplastic syndromes $[11,12]$. However, over $50 \%$ of renal masses are detected coincidentally, with only a few cases presenting with the classical symptoms mentioned above. Furthermore, the presence of these symptoms is often indicative of locally advanced or metastatic kidney cancer [13]. Imaging tests and renal biopsy, as well as laboratory tests like urinary cytology, are crucial methods to diagnose kidney malignancy [14]. However, imaging evaluation may sometimes lead to misdiagnosis when encountering rare diseases such as CD and IgG4-RD. They can not only pose a diagnostic challenge since these lesions masquerade as kidney malignancy, but also result in unnecessary radical nephrectomy, and delay appropriate treatment $[15,16]$. In fact, percutaneous needle core biopsy has been increasingly applied to guide clinical decisions in the management of renal masses, not only for its relatively high accuracy and safety in evaluating suspicious and undefinable masses, but also for avoiding unnecessary surgery [17-19]. Additionally, it is important to recognize that patients managing with nephrectomy, especially radical nephrectomy, have a high chance of developing renal insufficiency [20]. Impairment of kidney function after nephrectomy may eventually lead to chronic kidney disease. This is even more likely in patients who show a decline in GFR of 

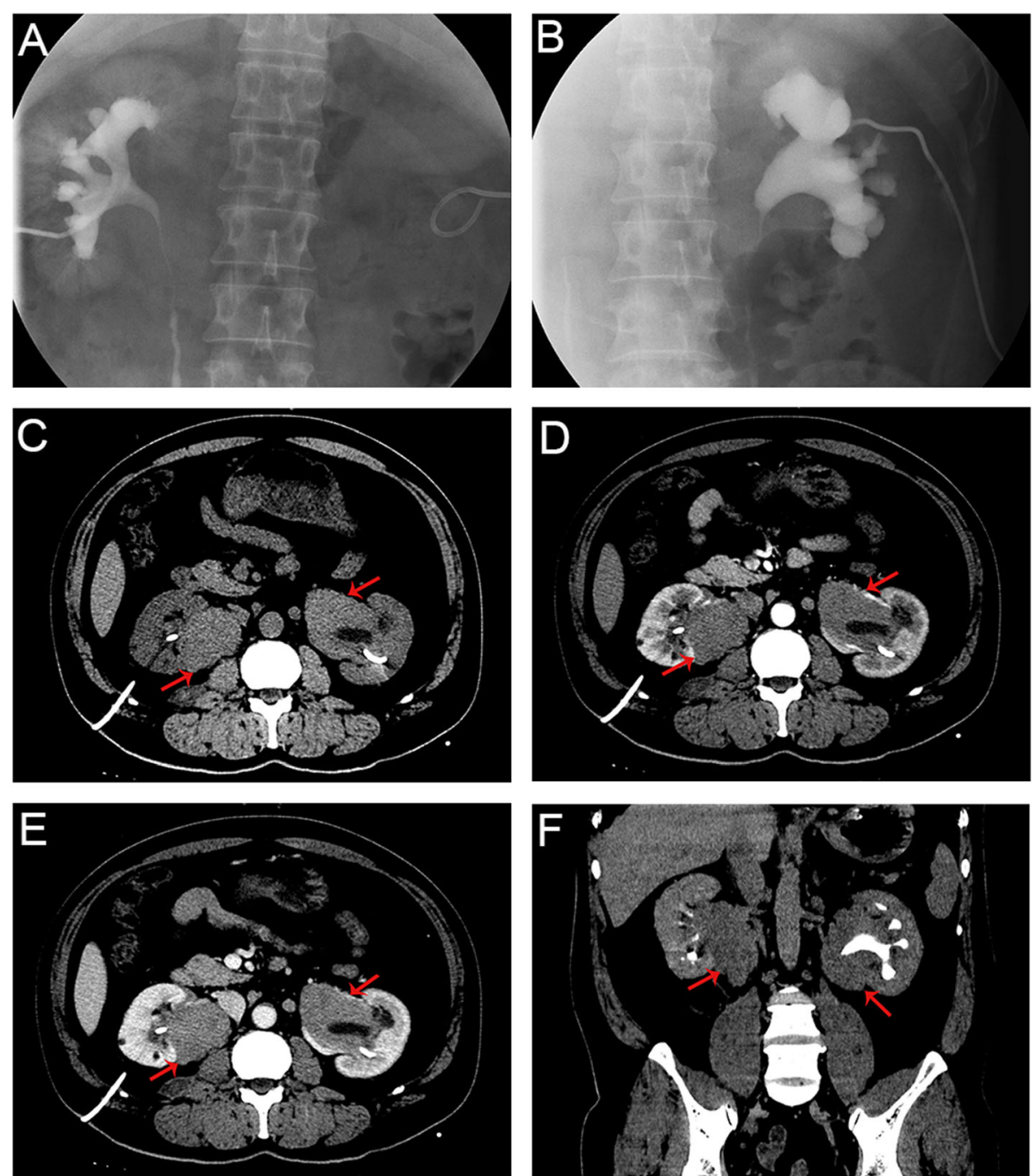

Fig. 3 The antegrade urography of the left (A) and right (B) kidney, and contrast-enhanced abdominal CT urography of the lesions (red arrow) during plain scan phase $(\mathbf{C})$, arterial phase (D), venous phase $(\mathbf{E})$ and delayed phase $\mathbf{( F )}$ in case 2

the other kidney before the surgery [21]. Thus, distinguishing these two diseases from kidney malignancy is urgently required.

$\mathrm{CD}$ is an atypical and rare lymphoproliferative disease originally described by Benjamin Castleman in 1956 [22]. The incidence rate of $C D$ is roughly $21-25$ per million person-years [23]. CD can manifest at any age with the peak age of onset between 30 and 50 years, without significant gender differences [24]. The role of HHV-8 and HIV in the pathogenesis of CD is well documented $[25,26]$. However, there was no evidence for HHV-8 and HIV infection in our patient. Clinically, CD can be divided into two subtypes: unicentric $C D$ (UCD) and multicentric $\mathrm{CD}(\mathrm{MCD})$, based on its involvement in a single lymph node or region of lymph nodes and multiple lymph nodes, respectively. UCD patients are typically diagnosed in those with compressive symptoms or enlarged lymph nodes that are found serendipitously. Most of them are asymptomatic with normal laboratory findings. In contrast, MCD patients often present with lymphadenopathy in more than a single lymph node station accompanied by fever, anemia, emaciation, liver and kidney dysfunction, and other systemic manifestations, including TAFRO syndrome (thrombocytopenia, ascites, reticulin fibrosis, renal dysfunction, and organomegaly) [27, 28]. Our PET/CT scan of the patient suggested MCD with the median SUV ${ }_{\max }$ between 2.4 and 3.3. Regardless of the type of $\mathrm{CD}$, the median $\mathrm{SUV}_{\max }$ is usually approximately $3-8$, whereas higher values would be suggestive of malignancy [29]. However, in our case, patient mainly presented with hematuria and lower back pain, whereupon preoperative MRI indicated occupying lesion in the renal pelvis. Due to the lack of specificity in imaging findings, it is difficult to distinguish them from 

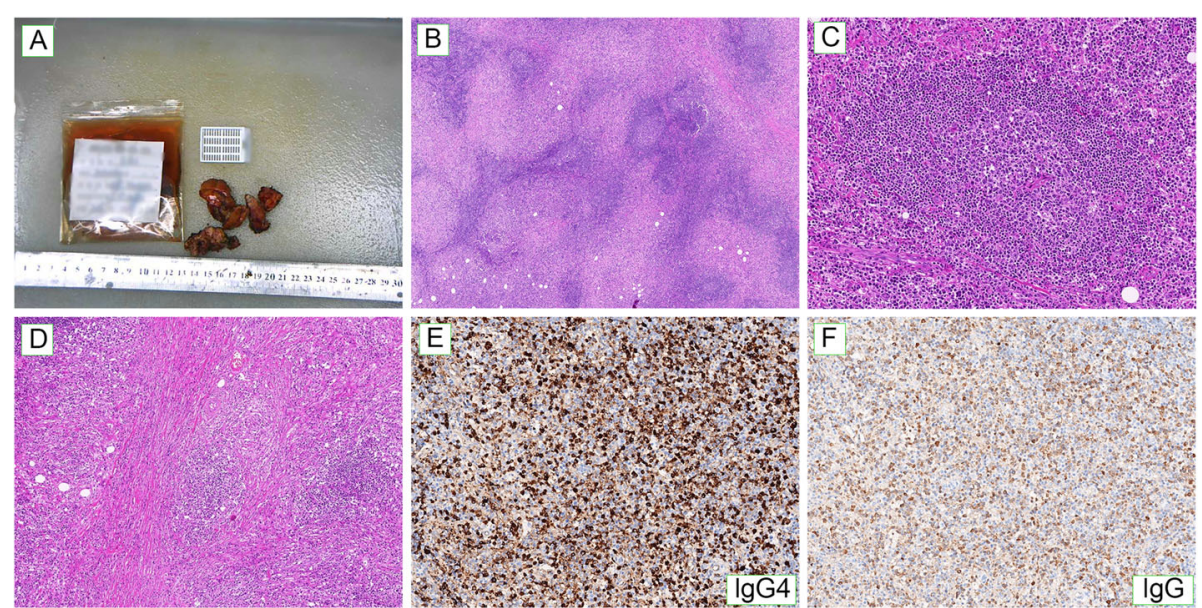

Fig. 4 Histopathologic features of the masses in case 2. (A) Gross observation of resected masses. (B) Increased degree of fibrosis and dense infiltration of plasma cells and lymphocytes in the specimen (40x). (C) Plenty of lymphocytes and plasma cells infiltrate and form germinal centers (200X). (D) Storiform-type fibrosis and obliterative phlebitis (100X). (E,F) Immunohistochemistry revealed many lgG (E) and lgG4 (F) positive plasma cells $(200 x)$

kidney malignancy by imaging alone. Furthermore, the postoperative histopathological result of $\mathrm{CD}$ is generally different from the preoperative imaging diagnosis [30]. Unfortunately, core needle biopsy was not taken to confirm the preoperative pathological diagnosis, resulting in subsequent radical nephrectomy in case 1 .

In fact, the diagnosis of $\mathrm{CD}$ depends entirely on histopathology. Histologically, $C D$ can be divided into three subtypes, namely, HV, PC an intermediate mixed type. The HV and PC types account for approximately 90 and $10 \%$ of $\mathrm{CD}$, respectively, whereas the mixed type is rare [31]. The pathology of the HV type is characterized by the proliferation of interfollicular capillaries and small hyaline vascular follicles. In contrast, PC type mainly manifests as the spreading of sheets of mature plasma cells in the interfollicular areas and hyperplastic germinal centers. The mixed type has morphological features of both types [4, 32]. Pathologically, the CD patient in our report was finally diagnosed as PC type with no significant systemic symptoms. The interfollicular zone was infiltrated by CD38 (+) and CD138 (+) plasma cells and amorphous eosinophilic deposition was observed in the germinal centers.

Generally, the treatment of UCD primarily based on surgical resection. Complete resection of involved lymphoid tissue is relatively recognized as the gold standard for management and can eliminate clinical symptoms with low recurrence [33]. Ten-year overall survival rate in UCD patients is approximately $90 \%$ [24]. Moreover, radiation therapy can be considered in those unsuitable for surgery [33]. Contrastingly, surgery is insufficient for the management of MCD with lower overall survival rate than UCD [24, 34]. The management of MCD is mainly based on glucocorticoids, chemotherapy, antiretroviral therapy, and monoclonal antibody therapy targeting IL-6 [33]. In case 1, after radical nephroureterectomy performed due to the misdiagnosis of $\mathrm{CD}$ as carcinoma of the renal pelvis, the patient underwent combination chemotherapy (R-COP) for the postoperative pathological diagnosis of $\mathrm{CD}$. Fortunately, no recurrence was observed in the follow-up PET/CT after chemotherapy.

IgG4-RD is a newly recognized chronic and progressive autoimmune disorder of unclear etiology, involving multiple organs and tissues. The disease responds well to glucocorticoids. It is characterized by elevated serum IgG4 concentrations and tumefactive lesions in the affected organs, caused by abundant infiltration of IgG4positive plasma cells and lymphocytes with fibrosis [3, 35]. The epidemiology of the disease remains poorly reported due to lack of recognition. However, Japan has estimated that the incidence of this disease would be $0.28-1.08 / 100,000$ population per year with the mean age of affected patients being 60 to 70 years [6]. IgG4$\mathrm{RD}$ exhibits more common in males with a male-tofemale ratio of 2 to 1 [36]. Clinical symptoms of IgG4$\mathrm{RD}$ vary depending on the involved organs, but most patients have relatively mild symptoms with a long disease course. Furthermore, IgG4-RD is likely to go undiagnosed or misdiagnosed because it is treated in different specialties depending on organ involvement [37]. In case 2 , our patient was sent to the urology department for anuria. Unlike other autoimmune diseases, systemic symptoms such as fever, malaise, swelling, and pain in joints are uncommon [38]. Hypergammaglobulinemia is frequently observed in laboratory testing of patients with IgG4-RD. Serology often reveals elevation in IgG, IgE, and eosinophil count [39]. Serum IgG4 $>135 \mathrm{mg} / \mathrm{dl}$ 
$(1.35 \mathrm{~g} / \mathrm{l})$ is the suggested cutoff value or diagnosis of IgG4-RD, with a sensitivity and specificity of 97.0 and $79.6 \%$, respectively [40]. However, we did not consider IgG4-RD as a diagnosis before surgery, and consequently, serum IgG4 concentration was not evaluated preoperatively. We then considered the mass lesions as lymphoma in CTU. Imaging of IgG4-RD patients usually reveals enlargement or compression of the different organs involved. Contrast-enhanced CT scans of IgG4RD with kidney involvement often presents multiple well demarcated low-density lesions in the kidney or thickening of the renal pelvis wall [37]. CTU in case 2 revealed large soft tissue lesions, but with mild-to-moderate homogeneous enhancement and smooth renal pelvis surface, which is a noteworthy imaging feature for urologists to identify kidney malignancy.

The diagnosis of IgG4-RD is primarily based on histopathological examination. Specific features include dense lymphoplasmacytic infiltrates in affected tissues or organs, obliterative phlebitis and storiform fibrosis [41]. Additionally, to diagnose IgG4-RD, the ratio of IgG4positive plasma cells to IgG-positive plasma cells should be greater than $40 \%$ in the affected tissue, with a sensitivity of $94.4 \%$ and a specificity of $85.7 \%$ [40]. However, increased IgG4-positive plasma cells is not a specific feature of IgG4-RD and can exhibited in various chronic inflammatory diseases like inflammatory bowel disease, vasculitis, and lymphoma, which do not exhibit histological features of storiform-type fibrosis and obliterative phlebitis [36]. In case 2, CD was diagnosed by intraoperative frozen section examination, but paraffin-embedded pathology and immunohistochemistry after surgery confirmed IgG4-RD. MCD is often difficult to distinguish from IgG4-RD since they have overlapping clinical and/ or histological features [42]. In 2011, the comprehensive clinical diagnostic criteria for diagnosis of IgG4-RD were established in Boston [43], which include [1] clinical manifestations of characteristic diffuse/localized enlargement or mass formation in single or multiple organs, [2] Serological tests, presenting elevated serum IgG4 levels $\geq 135 \mathrm{mg} / \mathrm{dl}$, and [3] histopathological features, showing dense infiltration of lymphocytes and plasma cells with fibrosis, and the ratio of IgG4-positive plasma cell / IgGpositive plasma cell $>40 \%$ and $>10$ IgG4-positive plasma cells per HPF. Only when all three criteria are met can a definitive diagnosis be made. A probable diagnosis can be made if conditions 1 and 3 are met, and 1 and 2 can make a possible diagnosis. In case 2 , we made a definitive diagnosis of IgG4-RD based on all three criteria above after surgery.

Once we confirm the diagnosis of IgG4-RD, glucocorticoids, a well-recognized therapy, can be considered for patients. Patients treated with glucocorticoids have an overall response rate and complete response rate of 93 and 66\%, respectively [36]. Combined use of immunosuppressants such as cyclophosphamide, mycophenolate mofetil, and azathioprine may be effective in the maintenance of remission, allowing for the reduction of steroids while helping prevent recurrence. In patients exhibiting glucocorticoid resistance or relapsing disease, rituximab is a better alternative [36, 44]. Moreover, urgent surgical intervention is needed to reduce the severity of obstruction symptoms and improve organ functions rapidly [44]. Our patient in case 2 responded well to glucocorticoids and cyclophosphamide after partial excision of the mass lesion. The follow-up imaging tests showed that drug therapy caused continued reduction in the size of the mass, indicating that the surgical excision may be unnecessary. Hence, we need to consider whether surgery for IgG4-RD can be avoided to reduce surgical risk and financial costs associated with overtreatment of benign conditions.

In conclusion, we reported two cases of two rare diseases, CD and IgG4-RD, presenting with renal spaceoccupying lesions and masquerading as kidney malignancy. The prominent feature in our cases is that preoperative diagnosis was difficult since $C D$ and IgG4-RD can exhibit nonspecific clinical manifestations and imaging findings, and our diagnosis was eventually based on postoperative pathology. Our cases highlight how $C D$ and IgG4-RD can affect the kidney, manifest as mass lesion, and mimic malignancy. Therefore, we recommend that clinicians should perform core biopsy while diagnosing patients with suspicious and undefinable renal masses to avoid unnecessary surgical treatment.

\section{Abbreviations \\ CD: Castleman disease; IgG4-RD: IgG4-related disease; CT: computed tomography; MRI: magnetic resonance imaging; $\mathrm{HV}$ : hyaline vascular; PC: plasma cell; PET/CT: positron emission tomography with computed tomography; R-COP: R = Rituximab; C: Cyclophosphamide; O: Vincristine (Oncovin); P: Prednisone; GFR: glomerular filtration rate; CTU: computed tomography urography; HPF: high powered field; HHV-8: human herpesvirus- 8; HIV: human immunodeficiency virus; UCD: unicentric Castleman disease;} MCD: multicentric Castleman disease

\section{Acknowledgements}

Not applicable.

\section{Authors' contributions}

$\mathrm{BL}$ and $\mathrm{YH}$ draft the manuscript and assisted with clinical data collection and interpretation. LT and JG performed the histological and

immunohistochemical examination. $\mathrm{XZ}$ and $\mathrm{HZ}$ contributed to the diagnosis and participated in the revised version. $X Z, H Z, B L, Y H$ performed surgery. All the authors approved the final version of the manuscript.

\section{Funding}

This work was supported by National Natural Science Foundation of China (No.81670688 and 81800666), Natural Science Foundation of Guangdong Province (No.2016A030313192, 2017A030310414 and 2018A0303130330), Medical Science and Technology Foundation of Guangdong Province (No.A2017365), and the Fundamental Research Funds for the Central Universities (No.19ykpy33). 


\section{Availability of data and materials}

Data are available on reasonable request from the corresponding author due to privacy or other restrictions.

\section{Declarations}

\section{Ethics approval and consent to participate}

The study involving human participants was reviewed and approved by Ethics Committee of the Third Affiliated hospital of Sun Yat-Sen University. Written informed consent was obtained from the participants for the publication of this report and any identifying images or information.

\section{Consent for publication}

We have fully obtained the patients' consent and there is no personal. information of the patients in our case report.

\section{Competing interests}

The authors declare that the research was conducted in the absence of any commercial or financial relationships that could be construed as a potential conflict of interest.

\section{Author details}

'Department of Urology, the Third Affiliated Hospital of Sun Yat-Sen University, Guangzhou, China. ${ }^{2}$ Department of Pathology, The Third Affiliated Hospital of Sun Yat-Sen University, Guangzhou, China.

\section{Received: 17 February 2021 Accepted: 28 July 2021}

\section{Published online: 10 August 2021}

\section{References}

1. Kay FU, Pedrosa I. Imaging of solid renal masses. Urol Clin North Am. 2018; 45(3):311-30. https://doi.org/10.1016/j.ucl.2018.03.013.

2. Simpson D. Epidemiology of Castleman disease. Hematol Oncol Clin North Am. 2018;32(1):1-10. https://doi.org/10.1016/j.hoc.2017.09.001.

3. Takahashi $H$, Yamamoto $M$, Suzuki $C$, Naishiro $Y$, Shinomura $Y$, Imai $K$. The birthday of a new syndrome: IgG4-related diseases constitute a clinical entity. Autoimmun Rev. 2010;9(9):591-4. https://doi.org/10.1016/j.autrev.201 0.05.003.

4. Dispenzieri A, Fajgenbaum DC. Overview of Castleman disease. Blood. 2020; 135(16):1353-64. https://doi.org/10.1182/blood.2019000931.

5. Dham A, Peterson BA. Castleman disease. Curr Opin Hematol. 2007;14(4): 354-9. https://doi.org/10.1097/MOH.0b013e328186ffab.

6. Umehara H, Okazaki K, Masaki Y, Kawano M, Yamamoto M, Saeki T, et al. A novel clinical entity, lgG4-related disease (IgG4RD): general concept and details. Mod Rheumatol. 2012;22(1):1-14. https://doi.org/10.1007/s10165011-0508-6.

7. Kamisawa T, Zen Y, Pillai S, Stone JH. IgG4-related disease. Lancet. 2015; 385(9976):1460-71. https://doi.org/10.1016/S0140-6736(14)60720-0.

8. Sato Y, Kojima M, Takata K, Morito T, Asaoku H, Takeuchi T, Mizobuchi K., Fujihara M., Kuraoka K., Nakai T., Ichimura K., Tanaka T., Tamura M., Nishikawa Y., Yoshino T. Systemic lgG4-related lymphadenopathy: a clinical and pathologic comparison to multicentric Castleman's disease. Mod Pathol 2009;22(4):589-599. doi:https://doi.org/10.1038/modpathol.2009.17.

9. Shephard E, Neal R, Rose P, Walter F, Hamilton WT. Clinical features of kidney cancer in primary care: a case-control study using primary care records. Br J Gen Pract. 2013;63(609):e250-5. https://doi.org/10.3399/bjgp13 X665215.

10. Bray F, Ferlay J, Soerjomataram I, Siegel RL, Torre LA, Jemal A. Global cancer statistics 2018: GLOBOCAN estimates of incidence and mortality worldwide for 36 cancers in 185 countries. CA Cancer J Clin. 2018;68(6):394-424. https://doi.org/10.3322/caac.21492.

11. Patard JJ, Leray E, Rodriguez A, Rioux-Leclercq N, Guille F, Lobel B. Correlation between symptom graduation, tumor characteristics and survival in renal cell carcinoma. Eur Urol. 2003;44(2):226-32. https://doi.org/1 0.1016/s0302-2838(03)00216-1.

12. Sacco E, Pinto F, Sasso F, Racioppi M, Gulino G, Volpe A, et al. Paraneoplastic syndromes in patients with urological malignancies. Urol Int. 2009:83(1):1-11. https://doi.org/10.1159/000224860.

13. Ignee A, Straub B, Schuessler G, Dietrich CF. Contrast enhanced ultrasound of renal masses. World J Radiol. 2010;2(1):15-31. https://doi.org/10.4329/wjr. v2.i1.15.
14. Ljungberg B, Albiges L, Abu-Ghanem Y, Bensalah K, Dabestani S, FernandezPello $S$, et al. European Association of Urology guidelines on renal cell carcinoma: the 2019 update. Eur Urol. 2019;75(5):799-810. https://doi.org/1 0.1016/j.eururo.2019.02.011

15. Hoshi A, Nitta M, Tokunaga M, Hoshino H, Hongo S, Hanai K, et al. Castleman's disease in the pararenal retroperitoneal space, which is indistinguishable from renal cell carcinoma: a case report. Hinyokika Kiyo. 2007;53(1):43-8.

16. Zhang H, Ren X, Zhang W, Yang D, Feng R. IgG4-related kidney disease from the renal pelvis that mimicked urothelial carcinoma: a case report. BMC Urol. 2015;15(1):44. https://doi.org/10.1186/s12894-015-0041-6.

17. Volpe A, Kachura JR, Geddie WR, Evans AJ, Gharajeh A, Saravanan A, et al. Techniques, safety and accuracy of sampling of renal tumors by fine needle aspiration and core biopsy. J Urol. 2007;178(2):379-86. https://doi.org/10.101 6/j.juro.2007.03.131.

18. Wang $X, L v Y, X u Z$, Aniu M, Qiu Y, Wei B, et al. Accuracy and safety of ultrasound-guided percutaneous needle core biopsy of renal masses: a single center experience in China. Medicine (Baltimore). 2018;97(13):e0178. https://doi.org/10.1097/md.0000000000010178.

19. Shen SS, Ro JY. Histologic diagnosis of renal mass biopsy. Arch Pathol Lab Med. 2019;143(6):705-10. https://doi.org/10.5858/arpa.2018-0272-RA.

20. McKiernan J, Simmons R, Katz J, Russo P. Natural history of chronic renal insufficiency after partial and radical nephrectomy. Urology. 2002;59(6):81620. https://doi.org/10.1016/s0090-4295(02)01501-7.

21. Huang WC, Donin NM, Levey AS, Campbell SC. Chronic kidney disease and kidney Cancer surgery: new perspectives. J Urol. 2020;203(3):475-85. https:// doi.org/10.1097/JU.0000000000000326.

22. Castleman BTV. CASE records of the Massachusetts General Hospital weekly Clinicopathological exercises: CASE 40011. N Engl J Med. 1954;250(1):26-30. https://doi.org/10.1056/NEJM195401072500107.

23. Munshi N, Mehra M, van de Velde H, Desai A, Potluri R, Vermeulen J. Use of a claims database to characterize and estimate the incidence rate for Castleman disease. Leuk Lymphoma. 2015;56(5):1252-60. https://doi.org/1 0.3109/10428194.2014.953145.

24. Talat N, Belgaumkar AP, Schulte KM. Surgery in Castleman's disease: a systematic review of 404 published cases. Ann Surg. 2012;255(4):677-84. https://doi.org/10.1097/SLA.0b013e318249dcdc.

25. Pria AD, Pinato D, Roe J, Naresh K, Nelson M, Bower M. Relapse of HHV8positive multicentric Castleman disease following rituximab-based therapy in HIV-positive patients. Blood. 2017;129(15):2143-7. https://doi.org/10.1182/ blood-2016-10-747477.

26. Siegel MO, Ghafouri S, Ajmera R, Simon GL. Immune reconstitution inflammatory syndrome, human herpesvirus 8 viremia, and HIV-associated multicentric Castleman disease. Int J Infect Dis. 2016:48:49-51. https://doi. org/10.1016/j.jijid.2016.05.005.

27. Talarico F, Negri L, lusco D, Corazza GG. Unicentric Castleman's disease in peripancreatic tissue: case report and review of the literature. G Chir. 2008; 29(4):141-4

28. Liu AY, Nabel CS, Finkelman BS, Ruth JR, Kurzrock R, van Rhee F, et al. Idiopathic multicentric Castleman's disease: a systematic literature review. Lancet Haematol. 2016;3(4):e163-75. https://doi.org/10.1016/S2352-3026(1 6)00006-5.

29. Oksenhendler E, Boutboul D, Fajgenbaum D, Mirouse A, Fieschi C, Malphettes $M$, et al. The full spectrum of Castleman disease: 273 patients studied over 20 years. Br J Haematol. 2018;180(2):206-16. https://doi.org/1 0.1111/bjh.15019.

30. Zhou YX, Ji Y, WU S. A CARE-compliant article: Unicentric Castleman disease presenting as a retroperitoneal mass of the upper edge of the pancreas: a case report. Medicine (Baltimore). 2020;99(11):e19515. https://doi.org/10.1 097/MD.0000000000019515.

31. Kim TU, Kim S, Lee JW, Lee NK, Jeon UB, Ha HG, et al. Plasma cell type of Castleman's disease involving renal parenchyma and sinus with cardiac tamponade: case report and literature review. Korean J Radiol. 2012;13(5): 658-63. https://doi.org/10.3348/kjr.2012.13.5.658.

32. Shahidi H, Myers JL, Kvale PA. Castleman's disease. Mayo Clin Proc. 1995 70(10):969-77. https://doi.org/10.4065/70.10.969.

33. Abramson JS. Diagnosis and Management of Castleman Disease. J Natl Compr Canc Netw. 2019;17(11.5):1417-9. https://doi.org/10.6004/jnccn.2019.5037.

34. Li Y, Zhao H, Su B, Yang C, Li S, Fu W. Primary hyaline vascular Castleman disease of the kidney: case report and literature review. Diagn Pathol. 2019; 14(1):94. https://doi.org/10.1186/s13000-019-0870-9. 
35. Stone JH, Zen Y, Deshpande V. IgG4-related disease. N Engl J Med. 2012; 366(6):539-51. https://doi.org/10.1056/NEJMra1104650.

36. Chen LYC, Mattman A, Seidman MA, Carruthers MN. IgG4-related disease: what a hematologist needs to know. Haematologica. 2019;104(3):444-55. https://doi.org/10.3324/haematol.2018.205526.

37. Kawano M, Saeki T. IgG4-related kidney disease--an update. Curr Opin Nephrol Hypertens. 2015;24(2):193-201. https://doi.org/10.1097/MNH. 0000000000000102.

38. Yamamoto M, Takahashi $\mathrm{H}$, Shinomura $\mathrm{Y}$. Mechanisms and assessment of IgG4-related disease: lessons for the rheumatologist. Nat Rev Rheumatol. 2014;10(3):148-59. https://doi.org/10.1038/nrrheum.2013.183.

39. Della Torre E, Mattoo H, Mahajan VS, Carruthers M, Pillai S, Stone JH. Prevalence of atopy, eosinophilia, and IgE elevation in IgG4-related disease. Allergy. 2014;69(2):269-72. https://doi.org/10.1111/all.12320.

40. Masaki Y, Kurose N, Yamamoto M, Takahashi H, Saeki T, Azumi A, et al. Cutoff values of serum IgG4 and histopathological lgG4+ plasma cells for diagnosis of patients with IgG4-related disease. Int J Rheumatol. 2012;2012: 580814-5. https://doi.org/10.1155/2012/580814.

41. Deshpande V, Zen Y, Chan JK, Yi EE, Sato Y, Yoshino T, et al. Consensus statement on the pathology of IgG4-related disease. Mod Pathol. 2012;25(9): 1181-92. https://doi.org/10.1038/modpathol.2012.72.

42. Arora K, Rivera M, Ting DT, Deshpande V. The histological diagnosis of lgG4related disease on small biopsies: challenges and pitfalls. Histopathology. 2019;74(5):688-98. https://doi.org/10.1111/his.13787.

43. Umehara H, Okazaki K, Masaki Y, Kawano M, Yamamoto M, Saeki T, et al. Comprehensive diagnostic criteria for lgG4-related disease (lgG4RD), 2011. Mod Rheumatol. 2012;22(1):21-30. https://doi.org/10.1007/s1 0165-011-0571-z.

44. Vasaitis L. IgG4-related disease: a relatively new concept for clinicians. Eur J Intern Med. 2016;27:1-9. https://doi.org/10.1016/j.jjim.2015.09.022

\section{Publisher's Note}

Springer Nature remains neutral with regard to jurisdictional claims in published maps and institutional affiliations.

Ready to submit your research? Choose BMC and benefit from:

- fast, convenient online submission

- thorough peer review by experienced researchers in your field

- rapid publication on acceptance

- support for research data, including large and complex data types

- gold Open Access which fosters wider collaboration and increased citations

- maximum visibility for your research: over $100 \mathrm{M}$ website views per year

At $\mathrm{BMC}$, research is always in progress.

Learn more biomedcentral.com/submissions 